\title{
AGE, SKILL, AND HAZARD PERCEPTION IN DRIVING
}

\author{
Avinoam Borowsky, David Shinar, and Tal Oron-Gilad \\ Ben Gurion University of the Negev \\ Beer Sheva, Israel \\ E-mail: Borowsky@bgu.ac.il
}

\begin{abstract}
Summary: Twenty-one young-inexperienced drivers (17-18 years old, 2.7 months of driving license), 19 experienced drivers (22-30, 7.3 years of driving license) and 16 elderly-experienced drivers $(65-72,37.5)$ observed six hazard perception movies (four movies included one planned hazardous event and two movies served as control) and were instructed to press a button each time they recognized a hazard. Participants' eye movements were recorded. Young drivers were the least sensitive in responding to immaterialized unplanned hazards, which occurred after the planned hazardous events. When the hazard was imminent, however, all drivers responded at the same time. Eye movement analysis revealed that all drivers detected the elements in the environment when they were salient, but gazing towards the right at T-intersections characterized only the more experienced drivers. The young drivers tended to gaze straight ahead. This study shows that experienced drivers learn to avoid hazards to which inexperienced drivers must respond. Responding to more hazardous situations, drivers' knowledge base expands, resulting in a more focused, goal-directed visual search and a higher sensitivity to potential hazards.
\end{abstract}

\section{INTRODUCTION}

The ability to identify hazardous situations while driving is an important skill, which enables the driver to overcome the complex cognitive demands that the traffic environment presents. This ability is known as hazard perception. Mills et al. (1998) defined hazard perception as the ability to read the road. Horswill and Mckenna (2004) added that hazard perception may be regarded as situation awareness for hazardous situations. Within this framework, novice drivers have difficulties in forming a holistic understanding of the current situation (e.g., Benda and Hoyos, 1983; Brown and Groeger, 1988; Armsby, Boyle and Wright, 1989).

According to Horswill and Mckenna (2004), from all the different components of driving skill, only hazard perception has been found to relate to traffic-accident involvement across a number of studies (e.g., Peltz and Krupat, 1974; Mckenna and Crick, 1991). Like any skill, it is reasonable to assume that drivers become better in the ability to detect hazards as their experience grows. Research has shown that novices are slower in detecting hazards, and they often recognize smaller numbers of hazards than experienced drivers. Chapman and Underwood (1998) found that the visual scanning patterns of experienced drivers appeared to adapt themselves to different road situations, while novice drivers tended to use the same scanning patterns for all road types. Also, novice drivers fixated for longer durations than experienced drivers. Much research has shown that feedback from the environment is the best traffic guide, and it improves hazard perception (e.g., Matthews \& Moran, 1986; Naatanen, and Summala, 1976). Experienced drivers who have experienced more hazardous situations acquire a much 
more well-established knowledge base than novices, and they search for hazards in a much more goal-directed and coherent manner. An established knowledge base allows them to identify, predict and avoid potential hazardous situations that may materialize. Young-inexperienced drivers with impoverished knowledge bases respond to materialized hazardous situations rather than predicting future hazardous situations. "They are helpless seekers of information within the environment" (Endsley, 1995). Indeed, some research has shown that experienced drivers are more afraid to miss a potential hazard than to maneuver in a discerned hazardous situation. For example, Armsby et al. (1989) asked participants to classify different traffic situation pictures, and found that fog was considered more hazardous among experienced drivers. In addition, Bragg and Finn (1986), who used a similar method, found that novices rated pedestrian episodes as more hazardous than experienced drivers but rated tailgating as less hazardous than experienced drivers. Furthermore, Benda and Hoyos (1983) found that novice drivers pay attention to unimportant details within the environment.

In contrast to young novice drivers, older drivers have well-established schemata that enable them to focus their attention on the appropriate information and to direct their attention according to a probabilistic information attitude. The schema is only minimally affected by aging, and in most cases remains intact (Bolstad and Hess, 2000).

In our study we presented six hazard perception movies to three driver's groups: 19 experienced drivers (22-30 years old), 21 novice drivers (17-18 years old), and 16 elderly drivers (65-72 years old). Participants were instructed to observe the movies as a driver driving down the road and to press a button each time they recognized a hazardous situation. When the first presentation of the movies ended, participants observed the movies again and had to classify them into an arbitrary number of groups according to the similarity of their hazardous situations.

\section{HYPOTHESES}

This research included three main hypotheses. First, elderly and experienced drivers will be more sensitive to potential hazards and will produce more button presses than young-inexperienced ones. Second, elderly and experienced drivers will fixate more towards hazardous potential locations whereas young-inexperienced drivers will scan the environment randomly and fixate only on salient objects. Third, old-experienced drivers and experienced drivers will be able to create a holistic representation of the environment whereas young-inexperienced drivers will pay more attention to unimportant details.

\section{METHOD}

\section{Participants}

Fifty-six participants-21 young-inexperienced drivers (17-18 years old, 2.7 months of driving license), 19 experienced drivers (22-30, 7.3 years of driving license) and 16 elderly-experienced drivers $(65-72,37.5)$ - participated as paid volunteers. All participants had normal vision, with uncorrected Snellen static acuity of 6/9 (20/30) or better, and normal color vision. The experienced drivers were students in Ben-Gurion University. Young-inexperienced drivers were 
recruited through driving schools, and the elderly drivers were usually retired people from the city of Beer - Sheva in Israel.

\section{Apparatus}

A 19" LCD screen with $1024 \times 768$ pixels, connected to a Pentium 4 personal computer was used in order to display the different movie scenes. The participant sat at an average distance of seventy centimeters from the LCD, creating a visual field of 22 degrees vertically and 26 degrees horizontally. The visual scan pattern was recorded with an eye tracking system (ETS) (Applied System Laboratories, Model 504), sampling the visual gaze at $50 \mathrm{~Hz}$, with a nominal accuracy of 1 degree. Six, 30- to 60-second driving scene movies were shown to the subjects. Four of the movies included planned hazardous situations and the other two were control. The six situations included (M1) braking of a lead car without signaling in order to park in a parking space, (M2) a control movie for M1 without braking of the lead car, (M3) a roller-skater entering the driving lane from the curb, (M4) bicycle veering into the center of the driving lane when the driver of a parked car suddenly opens the door to exit, (M5) braking of a lead car in response to a third driver suddenly entering from the right side of a $\mathrm{T}$ intersection, and (M6) control scene without any planned hazards. M1 and M2 where filmed in an urban area and the rest in a residential area. All movies were converted into a sequence of $760 * 576$ pixels single frames. A C++ program was used to send data to the ETS that indicated each frame switch. Each button press (utilizing a special button) was marked and indicated the subject's identification of a hazard situation. This procedure synchronized the eye movements with the appropriate frame.

\section{Procedure}

Participants were connected to the ETS after a short explanation of the experiment. The experiment was conducted in three parts, of which only the first two are discussed here. In the first part, the participants' gaze was calibrated and they observed two hazard perception movies and practiced with the experimental tasks. These movies were filmed in England and the subjects were reminded that they drive on the left side of the road. At the end of this part, the experimenter verified that the subject understood his or her task. In the second part participants were instructed to observe six hazard perception movies filmed from the driver's perspective in Israel. The order of the movies was randomized across subjects. Each participant was given Haworth et al.'s (2000) definition of a hazard. Participants were ordered to press a response button whenever they recognized a dangerous situation that required a preventive action. This procedure resembled Chapman and Underwood's (1998). After each movie presentation, the participant gave his or her opinion as to "what were the hazards in the movie you have just seen?" During the video presentations the participant's eye movements were recorded and synchronized with the button presses.

\section{RESULTS}

\section{Part 1: General between-movies analysis}

The first goal was to investigate whether movies 1, 3, 4, and 5 were indeed perceived as more hazardous than the control movies 2 and 6. A two-way ANOVA with repeated measures 
confirmed that the average number of button presses was significantly higher for the four planned hazardous scenes than for the two control scenes (1.65 versus 1.10 , respectively) $\left(\mathrm{F}_{1}\right.$, $\left.{ }_{53}=55, \mathrm{P}<0.001\right)$. The second goal was to examine whether fixations duration in urban areas (M1 and M2) were longer than in residential areas (M3-6), where the information rate per time unit is larger. A two-way ANOVA with repeated measures showed a significant main effect of road type, with average fixation durations of $229 \mathrm{~ms}$ for the urban scenes and $209 \mathrm{~ms}$ for the residential scenes $\left(\mathrm{F}_{1,36}=32.5, \mathrm{p}<0.001\right)$.

\section{Part 2: Specific within-movies events analysis}

We examined how drivers' experience relates to hazard perception. Drivers pressed the button not only in response to the real hazards perceived to be greatly hazardous but also in response to unplanned situations. Table 1 describes all events - planned and unplanned-according to the participants' responses. The bold entries represent the planned hazards.

Table 1. Events matrix

\begin{tabular}{|c|c|c|c|c|c|}
\hline \multirow{2}{*}{ Movie } & \multicolumn{5}{|c|}{ Event } \\
\hline & 1 & 2 & 3 & 4 & 5 \\
\hline 1 & First parking truck & Second parking truck & Red car brake & intersection & \\
\hline 2 & Passing lane & intersection & & & \\
\hline 3 & Old lady & Roller on curb & Roller on road & intersection & \\
\hline 4 & Bicyclist Recognition & Door's car opened & Bicyclist 2 & Children & Parking cars \\
\hline 5 & First intersection & White car brakes & White car turn right & Second intersection & \\
\hline 6 & First intersection & Car in front & Second intersection & After second intersection & \\
\hline
\end{tabular}

Table 1 shows that the participants detected 16 unplanned events as hazardous. The planned events, however, were the only events to which all drivers responded as hazardous.

The analysis included eye movement analysis and three dependent measures: reaction time, response sensitivity (button pressed or not), and classification type analysis (the verbal hazard instigator described by the subject). Events that most subjects thought were dangerous were analyzed in terms of the three dependent measures mentioned above. Events that were responded to by a minority of the participants were analyzed only in terms of eye movements and response sensitivity, and only if one driver group was more sensitive to them than the other two groups. Reaction time analysis demonstrated that when the hazards posed an immediate threat, all drivers responded equally fast. Response sensitivity analysis in movies with planned events revealed that young-inexperienced drivers were less sensitive in responding to potential hazards that did not materialize if they occurred immediately after the materialized hazardous situations that were planned. A two-way ANOVA with repeated measures showed two significant main effects, which included group type $\left(\mathrm{F}_{2,53}=4.078, \mathrm{P}=0.023\right)$ and the within-subjects factor before and after events $\left(F_{1,53}=12.405, P<0.001\right)$, as well as the interaction between these two variables $\left(F_{2}\right.$, $53=3.085, \mathrm{P}<0.054)$. Figure 1 presents the interaction. 


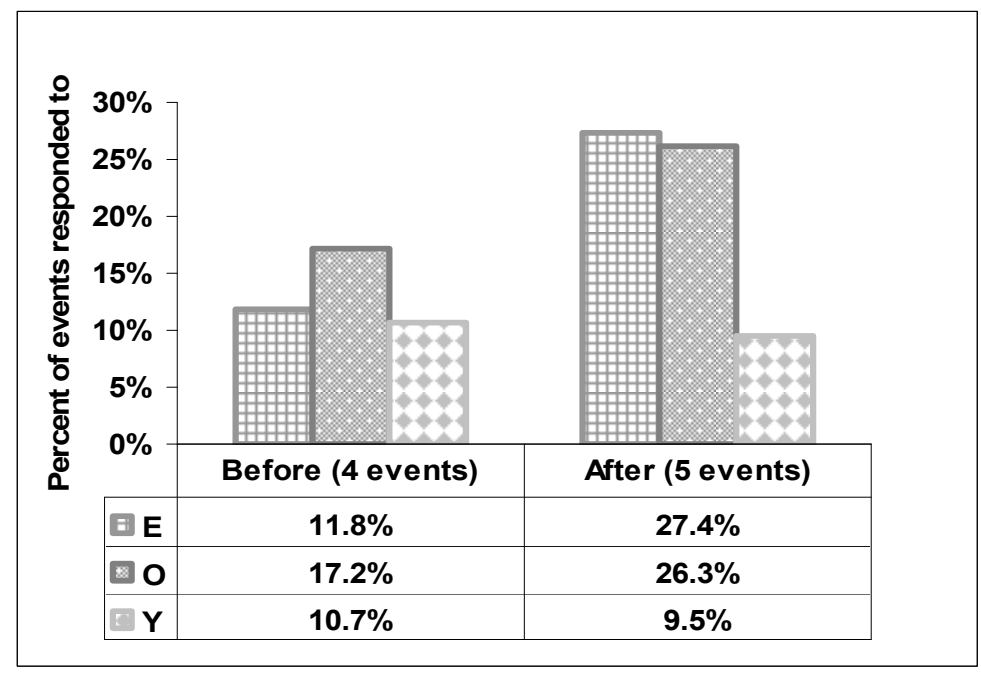

Figure 1. Percent of events responded to before/after planned-in-advance events

"Before" represents all 4 unplanned events that occurred before planned-in-advance events and "After" represents all 5 unplanned events, which occurred after the planned-in-advance events. For example, $11.8 \%$ is the average percent of events that the experienced drivers responded to from all 4 events that occurred prior to the planned-in-advance events. Before and after events included only episodes where the planned hazard instigator was absent from the scene.

Young-inexperienced drivers were much less sensitive and likely to respond than experienced and elderly drivers if a situation occurred after a planned event. Elderly drivers and experienced drivers were equally sensitive. The majority of these events included intersections and pedestrian walking on the curb. Eye movement analysis showed that all drivers detected the explicitly seen hazardous elements in the environment, but only the more experienced drivers were sensitive enough to respond to them. However, only the old-experienced $(\mathrm{O})(3$ out of 16 drivers [3/16] of all drivers who pressed the button in this event) and the young-inexperienced drivers $(\mathrm{Y})(0 / 21)$ were significantly different, while experienced drivers $(E)(2 / 19,40 \%)$ were not statistically different from either of these groups (O Vs E, $\mathrm{X}_{1}{ }^{2}=0.48, \mathrm{P}=\mathrm{NS}$; $\mathrm{Y}$ Vs O, $\mathrm{X}_{1}{ }^{2}=4.28, \mathrm{P}<0.05$; $\mathrm{E}$ $\left.\mathrm{Vs} \mathrm{Y}, \mathrm{X}_{1}{ }^{2}=2.33, \mathrm{P}<0.2\right)$. Figure 2 shows the fixations of the three driver groups superimposed on frame 500 .
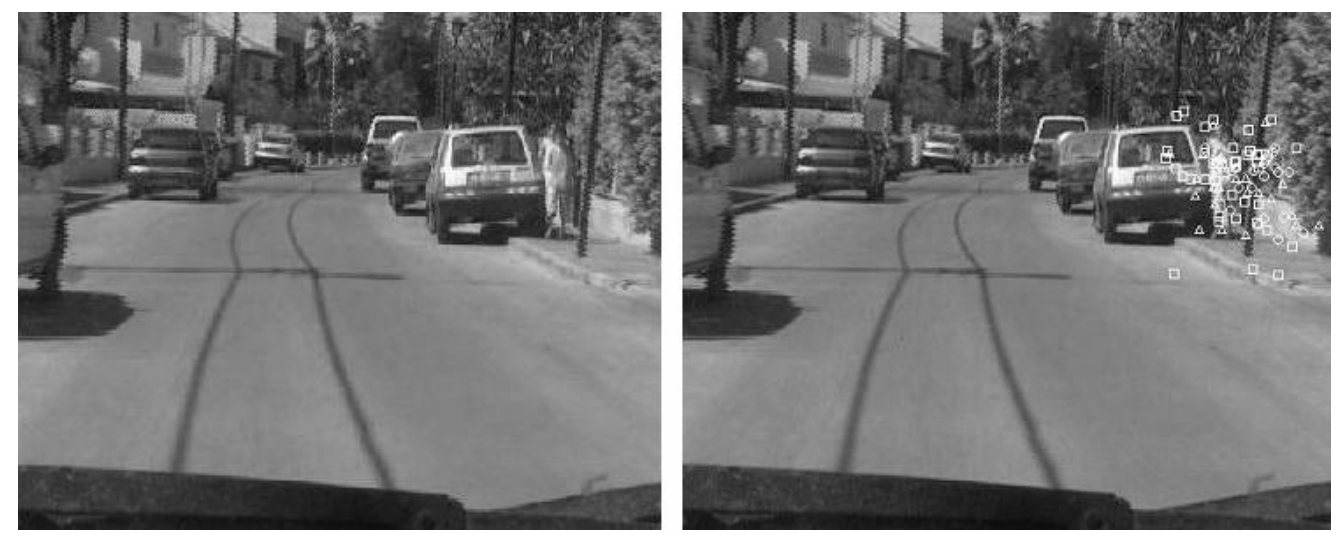

Figure 2. Event 4_4-Region of Interest fixation analysis 
In the figure, the white squares, triangles, and circles represent the fixations of young, experienced and elderly drivers, respectively. All fixations from frames 500-525 are superimposed on frame 500 .

When potential hazard situations such as T-intersections appeared, the more experienced drivers tended to gaze toward the right side where a road emerged, whereas young-inexperienced drivers gazed straight forward. Analyzing mean horizontal fixations location on frames 602-630 (road emerge from the right) in movie 3 revealed that both experienced drivers ( 16 drivers, Visual Degrees from the Center of the Road $(\mathrm{VDCR})=1.33)$ and old-experienced drivers $(11$ drivers, VDCR $=2.11$ ) tended to gaze toward the right side of the intersection while young-inexperienced drivers tended to gaze more straight forward (16 drivers, VDCR $=0.34)\left(\mathrm{F}_{2,40}=6.48, \mathrm{P}<0.004\right)$. Movie 5 analysis demonstrated similar statistical results. Figure 3 presents all fixations on frames 605-630 in movie 3 (left) superimposed on frame 605 and all fixations on frame 160-178 in movie 5 (right) superimposed on frame 170. The white squares, triangles, and circles represent the fixations of young, experienced and elderly drivers respectively. All fixations from frames 500-525 are superimposed on frame 500.
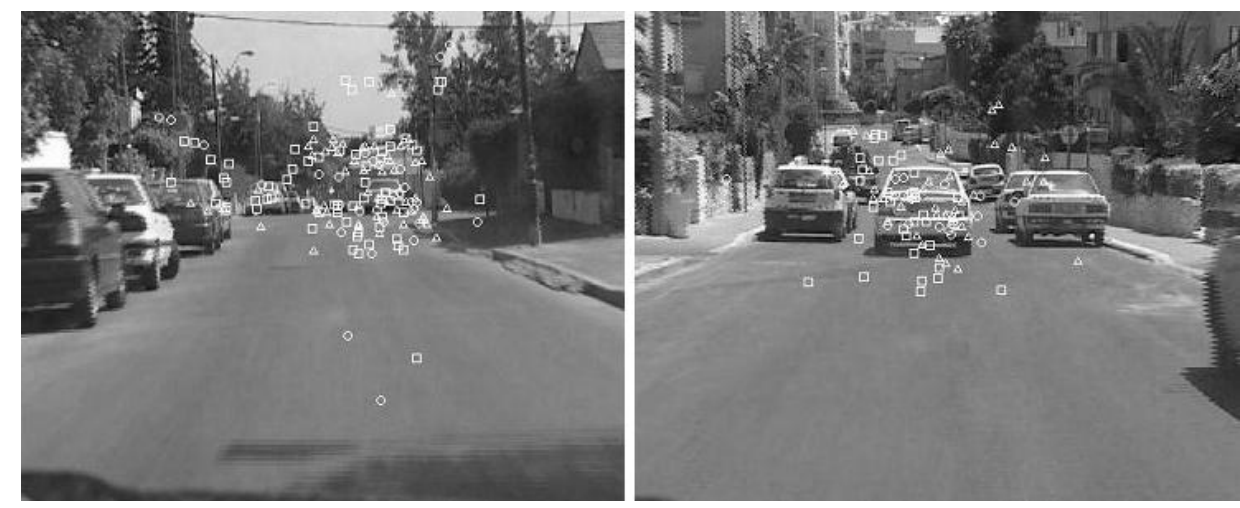

Figure 3. Visual scanning patterns at $\mathbf{T}$ intersections

\section{DISCUSSION}

This study shows that experienced drivers learn to avoid hazards to which inexperienced drivers must respond. The ability to perceive and recognize potential hazards before they become hazardous situations depends on past experience in similar situations where hazards materialized. Our findings and this conclusion are consistent with the results of Armsby et al. (1989) and Bragg and Finn (1986). Not having a sufficient experience-based knowledge base (e.g., Endsley, 1995) young-inexperienced drivers perceived the materialized hazards as extremely dangerous and ignored other less obvious hazards that appeared following the planned hazardous event. The experienced drivers on the other hand, perceived the level of hazardousness in a situation on the basis of their past experience in similar episodes, and therefore other events in which the hazard did not materialize were also considered hazardous. Furthermore, the more experienced drivers were more familiar with the environmental constraints (Vicente and Wang, 1998) and therefore they gazed towards locations where potential hazards were more likely to appear. These findings are consistent with Endsly's (1995) claim that novices may notice the same elements as experienced operators but might experience difficulties in integrating those elements into a holistic perception of the environment. 


\section{ACKNOWLEDGMENTS}

This research was partially supported by Ran Naor Foundation.

\section{REFERENCES}

Armsby, P., Boyle, A.J., and Wright, C.C. (1989). Methods for assessing drivers' perception of specific hazards on the road. Accident Analysis \& Prevention, 21, 45-60.

Benda, H.V., \& Hoyos, C.G. (1983). Estimating hazards in traffic situations. Accident Analysis \& Prevention, 15, 1-9.

Bolstad, A.C. and Hess M.T. (2000). Situation Awareness and Aging. Situation Awareness Analysis and Measurement, 277-302.

Brown, I.D., \& Groeger, J.A. (1988). Risk perception and decision taking during the transition between novice and experienced driver status, Ergonomics, 31, 587-597.

Chapman, P.R., and Underwood, G. (1998). Visual Search of Dynamic Scenes: Event Types and the Role of Experience in Viewing Driving Situations. Eye Guidance in Reading and Scene Perception, 369-393.

Endsley, M.R. (1995). Toward a theory of situation awareness in dynamic systems. Human Factors, 37, 32-64.

Finn, P., \& Bragg, B.W.E. (1986). Perception of the risk of an accident by young and older drivers. Accident Analysis \& Prevention, 18, 289-298.

Haworth, N., Symmons, M., \& Kowadlo, N. (2000). Hazard Perception By inexperienced Motorcyclist. Research Report, Monash University Accident Research Centre.

Horswill, M.S., and Mckenna, F.P. (2004). A cognitive approach to situation awareness: Theory and application. Aldershot; S.Banbury and S. Tremblay (eds.) Ashgate Publishing, 155-175.

Matthews, M.L., \& Moran, A.R. (1986). Age differences in male drivers' perception of accident risk: The role of perceived driving ability. Accident Analysis \& Prevention, 18, 299-313.

Mckenna, F.P., and Crick, J.L. (1991). Hazard perception in drivers: a methodology for testing and training. Final Report, Behavioural Studies Unit, Transport and Road Research Laboratory, Crowthorne, UK.

Mills, K.L., Hall, R.D., McDonald, M., \& Rolls, G.W.P. (1998). The effects of hazard perception training on the development of novice drivers skills. Report to Department Environment, Transport \& Regions (http://www.roads.detr.gov.uk/roadsafety/hazard).

Naatanen, R., and Summala, H. (1976). Road-user behavior and traffic accidents. Amsterdam: North- Holland.

Peltz, D.C., and Krupat, E. (1974). Caution profile and driving record of undergraduate males', Accident Analysis and Prevention, Vol. 6, 45-58.

Vicente, K.J., Wang, J.H. (1998). An ecological theory of expertise effects in memory recall. Psychological Review 105(1), 33-57. 